\title{
Reusability of eLearning Objects in the context of Learning Grids
}

\author{
Konrad Wulf \\ High Performance Computing Center Stuttgart (HLRS), Stuttgart, Germany \\ wulf@hlrs.de
}

\begin{abstract}
This paper examines the requirements for eLearning Object Metadata, in order to appropriately support pedagogic and economic goals as well as service oriented architectures like the Grid. The standard IEEE LOM is being tested against these requirements. In conclusion, it can be said that while current eLearning practices are well supported by the standard, the main insufficiencies concern a) the adequate description of ELOs that are services and not downloadable selfcontained programs and b) the commercial trading of ELOs.
\end{abstract}

Keywords: Reusability, eLearning, Standards, Metadata, eLearning Objects, Learning Grid, Grid Computing, requirements analysis, IEEE LOM, IMS Metadata

\section{INTRODUCTION}

The vision of an efficient and pedagogically effective European eLearning Grid Infrastructure, in my opinion, relies heavily on the reusability and - as a prerequisite - detectability of eLearning Objects. This is why this article takes a look at the requirements on the design of Metadata for such eLearning Objects. This analysis will then compare the requirements with a widely discussed standardization effort, namely IEEE LOM which is building on IMS Metadata and on work done by Ariadne and the Dublin Core Group.

\section{DEFINITION OF AN ELEARNING OBJECT (ELO)}

There are numerous definitions out there, but most of them have similar things in mind: eLearning Objects (ELOs) are chunks of teaching material that make sense on their own. The main purpose is the reuse in several different combinations within but also across organizational boundaries. This makes it sensible to include in the definition of an ELO the fact that it is tagged with meta information about it, so that it can be found by search engines. This is inline with the practical definition of Muzio et al. and others (see [5]). The aggregation level of an ELO can range from a "learning unit" to a "set of courses that lead to some kind of certificate".

Another important question is, whether an ELO should be considered as a downloadable thing or as a service. As the new generation of Grids is based on service oriented architectures (see e.g. [2]), it makes sense to regard the ELO as a service as the more general case whereas the downloadable thing - that is only executed on the client machine with no interaction with servers during execution time - represents one special case of it. The more general case will be used as working definition within this paper.

\section{REQUIREMENTS FOR REUSABLE ELEARNING OBJECTS IN A LEARNING GRID}

\subsection{Pedagogic point of view}

From a pedagogical perspective, the perfect eLearning process would be 1. responsive, 2. ubiquitous, 3 . collaborative, 4. experiential, 5. contextualised and personalised.

Responsiveness is a basic requirement that is valid for all Software, but especially on distributed systems like the Web, delay and also reliability are important Quality of Service (QoS) issues that need to be taken care of in order to deliver good eLearning services. Latency in delivery of requested learning resources highly influences a learner's motivation (see [6]). In many occasions, among other factors, the server side limitations lead to delay (see [7]). So for an ELO with service character, information about the current server performance situation and how it is influenced by the execution of another instance of the ELO is valuable for determining not only the technical, but also the motivational quality of an ELO (R_ServicePerformance). This topic is closely related to the monitoring of Service Level Agreements (SLA), which is a big issue of its own. So in terms of practicability this information will have to be concise. 
Ubiquity stands for the wish for accessibility at any time and from anywhere using a variety of devices. Ubiquity is potentially a central advantage of eLearning compared to classical class room teaching, as it enables new organisational patterns of learning. This implies that interoperability of protocols and data formats is essential, stressing also the need for standardized metadata.

Collaboration and group working can be very beneficial for the motivation of the learner. The sense of community, as well as the pure possibility to discuss open questions improves the performance of the learner, may it be that there is a mentor or may it be that there are fellow learners (see [8], pp.187-206). To natively integrate a collaboration tool, it seems sensible to specify such a thing in the metadata of an ELO - perhaps not on learning unit level, but surely on course level or higher. In the simplest case a chat room could be stated, or a mailing list (R_IntegratedCollaboration).

Experiential: The learning material should be highly interactive, so that the learner can make experiences through Learning by Doing (see [8], pp. 13-26). The learner should not only read texts (=> information transfer paradigm), but also use the information in a productive way by fulfilling some task so that he really understands. Thus, this requirement asks for possibilities of direct manipulation, feedback and the possibility of making mistakes. A requirement for ELO metadata derived from this is that we need some standardized indication of interactivity of a particular ELO (R_InteractivityDegree).

Contextualization and Personalization seems to be the most challenging of the requirements. It means that the learning system needs to know about the pre-knowledge of the learner, his ways and speed of learning etc. So once the Learning Management System (LMS) knows these details about the learner, how does its search engine find suitable ELOs? The answer is that metadata should contain information on the progression pace (R_ProgressionPace) and the assumed pre-knowledge (R_AssumedPreknowledge).

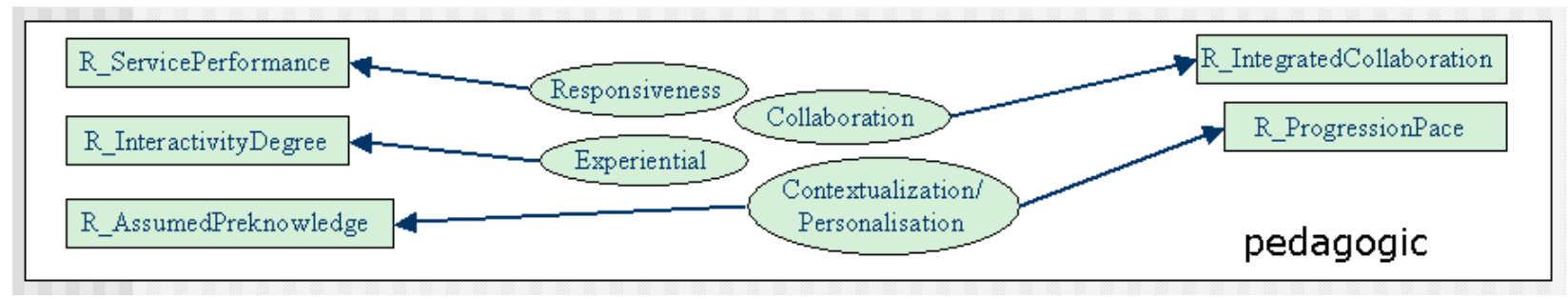

FIGURE 1: Requirements resulting from pedagogical goals

\subsection{Economic point of view}

From an economic and enterprise point of view, the eLearning content should also be 1. cost-efficiently producible, 2. findable and 3. saleable.

Cost-efficiency: Cost-efficiency can be reached through either a) reducing the costs of production or b) through increasing the benefits. Concerning a) it can be said that up to now, the production is still very costly, as it usually implies several specialists working together: a didactical expert, a content expert and a programmer. In order for eLearning to be successful, it should be possible for a content expert to produce an ELO alone through appropriate tool support. Here, the description of the metadata of an ELO doesn't seem to influence this. But concerning b) metadata as such do increase the benefits by making reuse more probable. Depending on the design of the metadata, configurability, i.e. that the ELO can switch to different languages, different difficulty levels, allow input/ouput data etc. would additionally foster reuse (R_Configurability, see [1], pp. 8-12). Saleability, discussed below, can additionally increase the benefits that are achievable.

Findability: This requirement asks for ease of retrieving an ELO of the right quality. For Standard text formats such as ASCII, doc and PDF files searching is comparatively easy and good search engine have meanwhile been developed. The quality is thereby determined by popularity in terms of amount of referring links etc. But many pedagogically valuable ELOs are usually in a binary format, so good findability can only be achieved by standardized metatagging.

To determine the quality of an ELO, a Learning Management System might want to know the source of an ELO, as this can be an indicator. An ELO offered by MIT for example would be considered of higher quality than one offered by some unknown university (R_Originlssuinglnstitution). Different cultures also might have different 
understandings of particular subjects, so that another indicator for quality can be the country the ELO originates from (R_OriginCountry). Furthermore, an important factor in reality for signalling quality is certification. Also certification should be possible for ELOs (R_Certification), because we need a means of judging the overall quality of the content - whether the content is actually considered to be true, how well it is made etc. ELO meta information can be more reliable when certified by a third party authority.

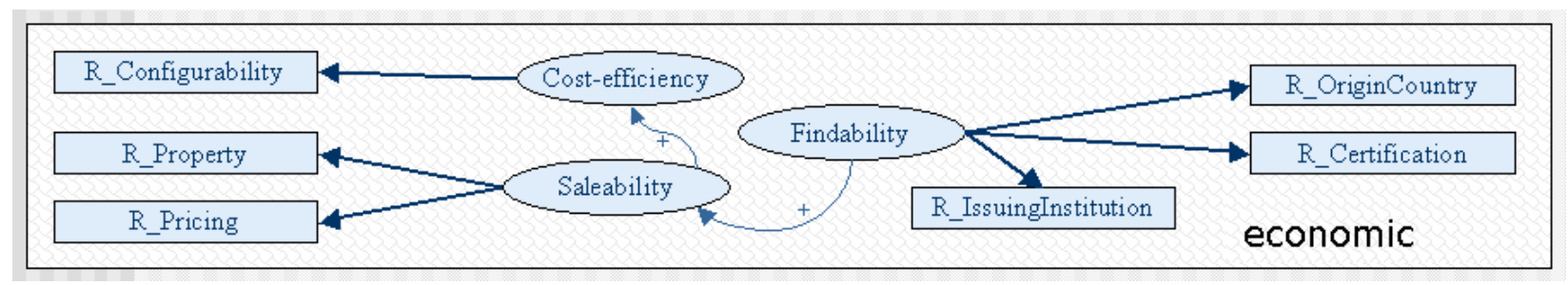

FIGURE 2: Economic requirements for ELO metatagging

Saleability: The requirement that an eLearning Object should be sellable in order to open up electronic market places, implies that it should be possible to protect intellectual property in the first place (R_Property, see also [5], p.7). This could be done for example by stating the copyright owner and sheltering the metadata from manipulation through e.g. encrypted checksums or watermarking. Moreover, it would be good if these eLearning Objects could already be labelled with a price, just like it can be encountered in the supermarket. Then a smooth pay by use via micro payment or similar could be used when the ELO is being requested and consumed in an open marketplace (R_pricing). It is worth noting that a free ELO is just a special case (price $=0$ ) of the more general case (price $>=0$ ). Findability leverages saleability.

\subsection{Limits to reusability}

Here, some general remarks about the limits to reusability will be made. These limits lead directly to some more requirements for the metadata.

First of all, the content that is to be delivered is nearly always tied to a language, so it is language specific and needs to be localized in order to be used in other language areas. This leads to the requirement $R$ _Language.

Then an eLearning Object, if it is not static plain text only, has some data format that requires a complementary application in a particular version to access the content. This means the metadata need to specify necessary software (R_ReqSoftware).

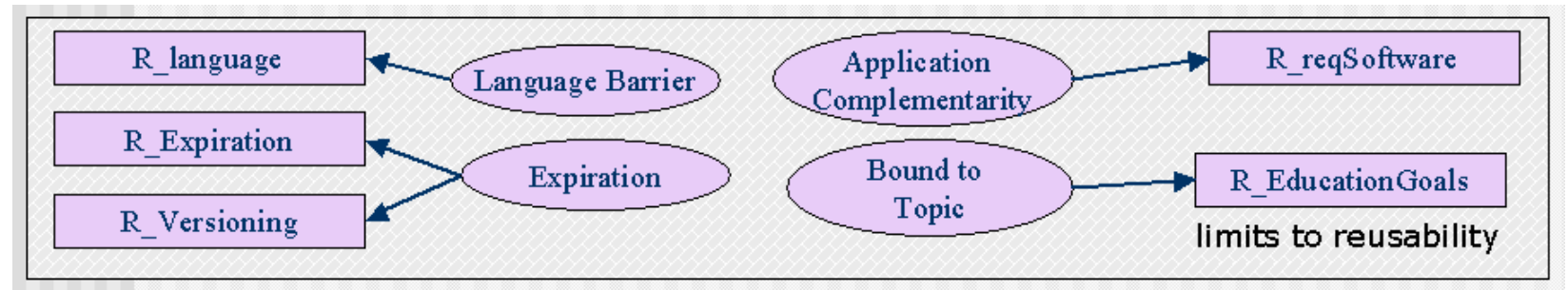

FIGURE 3: Limits to reusability of elearning objects

ELOs are more or less time-bound. Sooner or later the learning material will not be "safe to use" anymore. This could be for technical reasons or because the contents simply is not valid or of interest anymore. Such an expiration often cannot be forecasted. But there are some second-best indicators like the date of creation (R_Expiration) and whether a new version exists of the same ELO (R_Versioning).

An at first sight trivial limit for reuse is, of course, that the topic of the ELO is only valuable if it serves a particular educational goal. But how do we know that without looking into the ELO itself? There must be some specification of the educational goals that the ELO helps to reach. A short description of the goals would help (R_EducationalGoals).

\section{ANALYSIS OF THE EXISTING STANDARD IEEE LOM}

The IEEE Standard draft LOM is a unification effort of the US funded IMS and the EU funded ARIADNE project to merge and correlate their efforts in standardizing the metadata description of eLearning Objects (see[4] and [3]). LOM is very similar to IMS Metadata. For the overall structure of IMS metadata and IEEE LOM that is being referred to, see Figure 4 in the Appendix. 
Find below the results of the evaluation, in which IEEE LOM has been checked against the requirements that have been derived above:

\begin{tabular}{|c|c|c|}
\hline Requirement & Judgement & Grp. \\
\hline R_InteractivityDegree & $\begin{array}{l}\square 5.1 \text { Interactivity Type, } 5.2 \text { Learning Resource Type and } 5.3 \\
\text { Interactivity Level together give a pretty good idea of the degree of } \\
\text { interactivity of the ELO, using a closed and therefore well } \\
\text { comparable taxonomy. }\end{array}$ & \multirow{5}{*}{ 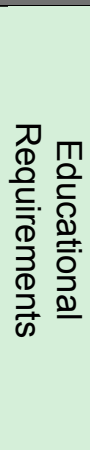 } \\
\hline R_ProgressionPace & $\begin{array}{l}\square 5.4 \text { Semantic Density ( } 5 \text { points linear scale), together with 5.6- } \\
5.8 \text { should make it easy for search engines to find an ELO } \\
\text { according to a learner's progression pace. }\end{array}$ & \\
\hline R_AssumedPreknowledge & $\begin{array}{l}\square \text { 5.6 Context, 5.7 Typical Age Range, 5.8 Difficulty indicate the } \\
\text { target audience using precise values out of an enumeration. }\end{array}$ & \\
\hline R_ ServicePerformance & 冈 In 4 Technical, no suitable entry space is designated. & \\
\hline R IntegratedCollaboration & 冈 In 4 Technical, no suitable entry space is designated. & \\
\hline R_Property & $\begin{array}{l}\text { No answer to the difficult question as to how to protect the } \\
\text { property from being copied and manipulated. In } 6.2 \text { and } 6.3 \text { it is } \\
\text { possible to specify copyright and license conditions, but there are } \\
\text { no standard license terms to choose from and a search engine will } \\
\text { have a hard time isolate the copyright holder from the free text } \\
\text { merged containing license conditions and the copyright holder. }\end{array}$ & \multirow{11}{*}{ 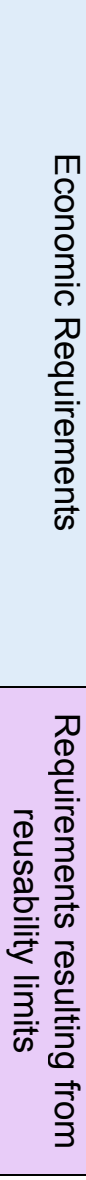 } \\
\hline R_Pricing & $\begin{array}{l}\text { } 6.1 \text { Cost specifies only whether the use (what kind of usage?) } \\
\text { will be for free or not. Why not specify the price right away, with } \\
\text { price=0 being "no cost"? }\end{array}$ & \\
\hline R_Issuinglnstitution & $\begin{array}{l}\square 凶 2.3 \text { Contribute and } 6.3 \text { Description will give a pretty good } \\
\text { impression about the source of an ELO, although it might not be } \\
\text { concise enough. }\end{array}$ & \\
\hline R_OriginCountry & $\begin{array}{l}\square \otimes \text { is NOT an extra item with enumeration values, but usually to } \\
\text { be read (between the lines) from } 2.3 \text { Contribute }\end{array}$ & \\
\hline R_Certification & $\begin{array}{l}凶 \text { there is no slot for third party certification Information for } \\
\text { indication of good quality }\end{array}$ & \\
\hline R_Configurability & 冈 In 4 Technical, no suitable entry space is designated??? & \\
\hline R_Language & $\square$ 1.3 Language: following ISO 639:1988 and ISO 3166-1:1997. & \\
\hline R_ReqSoftware & $\begin{array}{l}\square 凶 4.1 \text { Format in MIME type and } 4.4 \text { Requirement defines } \\
\text { necessary other resources, but there is no unique taxonomy for } \\
\text { these. }\end{array}$ & \\
\hline R_Expiration & $\begin{array}{l}\square \bowtie \text { 2.3.3 Date helps judging the "freshness", but does not help } \\
\text { distinguishing between more or less durable ELO content. }\end{array}$ & \\
\hline R_Versioning & $\begin{array}{l}\square 2.1 \text { Version allows for a check on whether you are working with } \\
\text { the latest version }\end{array}$ & \\
\hline R_educationGoals & $\begin{array}{l}\square 1.4 \text { Description: this is primarily for the user to judge, but no } \\
\text { standard taxonomy is being used. } \\
1.5 \text { Keyword }+1.6 \text { Coverage: this together with } 1.4 \text { can give } \\
\text { indications for the search engine. }\end{array}$ & \\
\hline
\end{tabular}

TABLE 1: Checklist for IEEE LOM metadata format

The checklist in Table 1 shows the results of testing LOM against the requirements. In summary, it can be said that the educational needs can be met to a wide extent using this specification. Those "educational" requirements that are not fulfilled come down to rather technical specifications that are missing and arise when ELOs are being thought of as services. The limits for reusability can also be told fairly well, although it would be desirable that the required resources (4.4) would be supplied in a more standard way (enumeration), in order to be better comparable. Also, judging the validity of content in terms of time - whether an ELO is outdated - still is an issue that is not really satisfactory solved (due to the nature of the problem). R_Configurability, here derived from economic goals, could also be seen as a limit for reusability. This limit could be widened up by allowing entries about setup choices and interfaces, which hasn't found its way into the specification, yet. 
What is not adequately addressed at all, are some basic economic requirements, if the possibility of an electronic market on the level of ELOs shall be opened up through using this standard. The usual indicators for high quality can not or only insufficiently be provided. Then the problem of protection of intellectual property is not even being addressed. Finally, and this is quite simple, there is no slot allocated for specifying prices for different kinds of usages.

\section{CONCLUSION}

As this analysis has shown, the IEEE Standard LOM addresses many requirements appropriately and therefore is a good starting point for the development of eLearning Grids. But some major improvements will have to be made concerning the support of delivery of high quality ELO services. Moreover, if the ELO material is to be bought and sold, usually across organizational entities, the specification really needs some profound improvement, regarding the protection of property, pricing, definition of usage patterns / standard licenses, and signalling of certified quality.

\section{REFERENCES}

[1] El Saddik, A. et al. (2001). Reusability and Adaptability of Interactive Resources In Web-Based Educational Systems. ACM Journal of Educational Resources in Computing, 1(1), Article \#4, 1-19 (http://portal.acm.org/citation.cfm?id=376699).

[2] Foster, I. et al. (2002). Grid Services for Distributed System Integration. Computer, 35(6), 37-46. (http://www.globus.org/research/papers/ieee-cs-2.pdf)

[3] IMS Global Learning Consortium, Inc. (2001). IMS Learning Resource Meta-Data Information Model - Version 1.2.1 Final Specification. (http://www.imsglobal.org/metadata/)

[4] Learning Technology Standards Committee of the IEEE (2002). Draft Standard for Learning Object Metadata. (http://lsc.ieee.org/wg12/files/LOM $1484121 \mathrm{v} 1$ Final Draft.pdf)

[5] Muzio, J. et al. (2001). Experiences with Reusable eLearning Objects: From Theory to Practice. Centre for Economic Development and Applied Research (CEDAR), Royal Roads University, Victoria, BC, Canada. (http://www.cedarlearning.com/CL/elo/eLearningObjects sml.pdf)

[6] Ramsay, J., A. Barbesi, and J. Preece, (1998) A psychological investigation of long retrieval times on the World Wide Web. Interacting with Computers, 10(10), 77-86.

[7] Ruddle, A. et al.(2002). Determining the Sources of Delay in a Distributed Learning Environment. Proceedings of 1st LEGE-WG International Workshop on Educational Models for GRID Based Services, Lausanne, CH, 16 September, eWiC (http://ewic.bcs.org/conferences/2002/1stlege/session3/paper2.htm).

[8] Schank, R. (2001). Designing World-Class E-Learning: How IBM, GE, Harvard Business School, And Columbia University Are Succeeding At E-Learning. McGraw-Hill, New York 


\section{ANNEX}

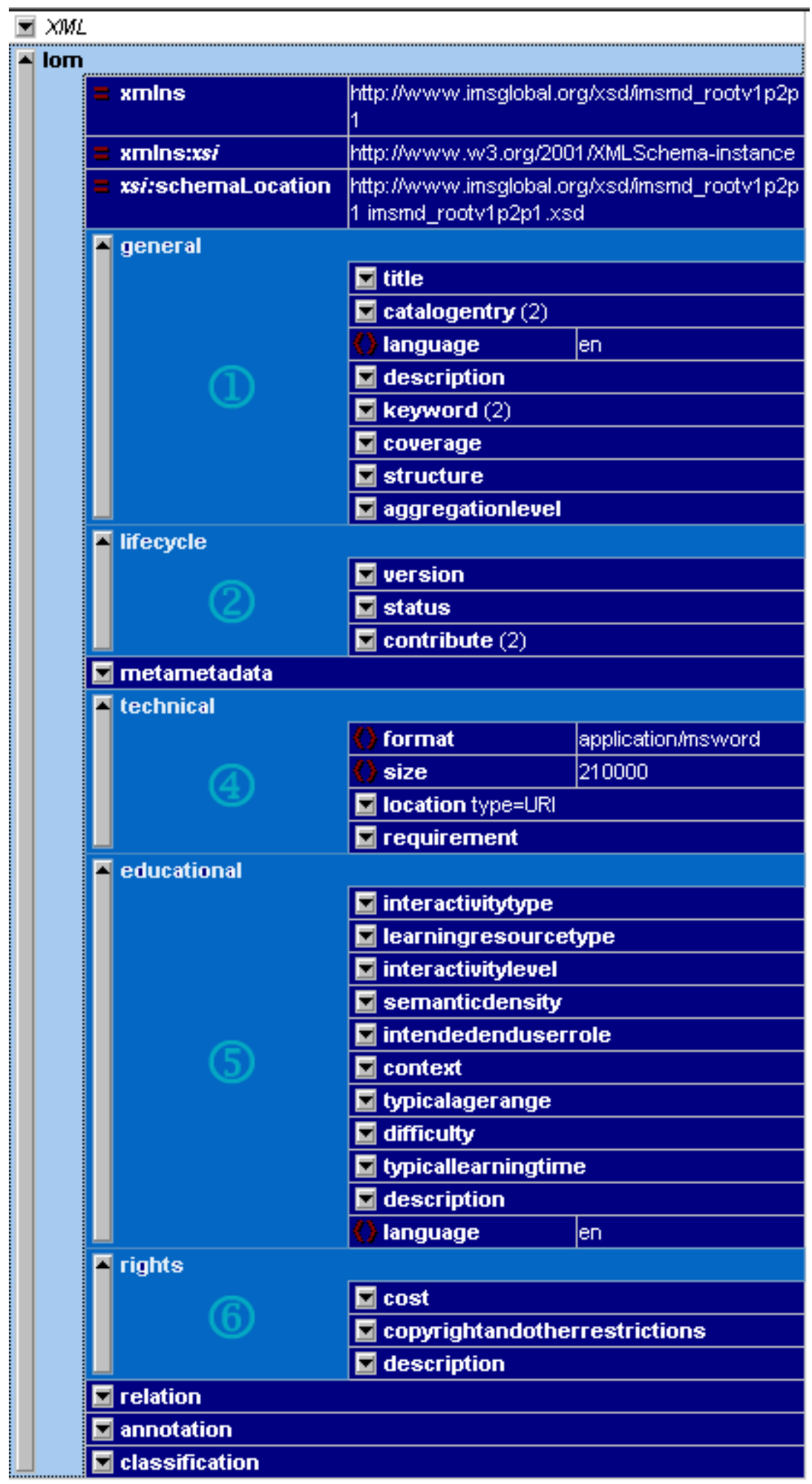

FIGURE 4: the overall structure of IMS METADATA / IEEE LOM 